\title{
Systematic analysis of the relationship between ovarian cancer prognosis and alternative splicing
}

\author{
Di Zhang, Dan Zou, Yue Deng and Lihua Yang ${ }^{*}$ (D)
}

\begin{abstract}
Background: Ovarian cancer(OC) is the gynecological tumor with the highest mortality rate, effective biomarkers are of great significance in improving its prognosis. In recent years, there have been many studies on alternative splicing (AS) events, and the role of AS events in tumor has become a focus of attention.

Methods: Data were downloaded from the TCGA database and Univariate Cox regression analysis was performed to determine AS events associated with OC prognosis.Eight prognostic models of OC were constructed in R package, and the accuracy of the models were evaluated by the time-dependent receiver operating characteristic (ROC) curves.Eight types of survival curves were drawn to evaluate the differences between the high and low risk groups. Independent prognostic factors of $\mathrm{OC}$ were analyzed by single factor independent analysis and multi-factor independent prognostic analysis.Again, Univariate Cox regression analysis was used to analyze the relationship between splicing factors(SF) and AS events, and Gene Ontology(GO) and Kyoto Encyclopedia of Genes and Genomes(KEGG) enrichment analysis were performed on OS-related SFs to understand the pathways.
\end{abstract}

Results: Univariate Cox regression analysis showed that among the 15,278 genes, there were 31,286 overall survival (OS) related AS events, among which 1524 AS events were significantly correlated with OS. The area under the time-dependent receiver operating characteristic curve (AUC) of AT and ME were the largest and the RI was the smallest,which were 0.757 and 0.68 respectively. The constructed models have good value for the prognosis assessment of OC patients. Among the eight survival curves, AP was the most significant difference between the high and low risk groups, with a $P$ value of $1.61 \mathrm{e}-1$. The results of single factor independent analysis and multi-factor independent prognostic analysis showed that risk score calculated by the model and age could be used as independent risk factors.According to univariate COX regression analysis, 109 SFs were correlated with AS events and adjusted in two ways: positive and negative.

Conclusions: SFs and AS events can directly or indirectly affect the prognosis of OC patients. It is very important to find effective prognostic markers to improve the survival rate of OC.

Keywords: Ovarian cancer, Alternative splicing, Survival model, Prognosis, Splicing factors

\section{Introduction}

For humans, all the important components of the body need proteins, and the diversity of proteins provides the

*Correspondence: 13759481789@163.com

Department of Gynaecology, the 2nd Afliated Hospital of Kunming

Medical University, Kunming, Yunnan, China basis for the diversity of body functions. AS as an important mechanism for post-transcriptional regulation of gene expression, which can produce the same, similar or even opposite proteins and provide a basis for the expansion of functional proteome diversity in eukaryotic genes when acts on precursor messenger RNA (pre-mRNA) [1]. AS events occur between $92 \%$ and $94 \%$ of human original author(s) and the source, provide a link to the Creative Commons licence, and indicate if changes were made. The images or other third party material in this article are included in the article's Creative Commons licence, unless indicated otherwise in a credit line to the material. If material is not included in the article's Creative Commons licence and your intended use is not permitted by statutory regulation or exceeds the permitted use, you will need to obtain permission directly from the copyright holder. To view a copy of this licence, visit http://creativecommons.org/licenses/by/4.0/. The Creative Commons Public Domain Dedication waiver (http://creativeco mmons.org/publicdomain/zero/1.0/) applies to the data made available in this article, unless otherwise stated in a credit line to the data. 
genes, a higher proportion than many other species and exactly in line with the fact that humans have more complex morphology and behavior [2]. Many studies have shown that AS events related to the initiation and progression of many diseases, from the initial research in the Mediterranean anemia and spinal muscular atrophy, in recent years glioblastoma, clear cell carcinoma, renal cell carcinoma and hepatocellular carcinoma (HCC) and so on many kinds of cancer are discussed [3-6]. More and more studies have shown that AS can affect the progress of cancer through multiple biological processes such AS cell proliferation, apoptosis, tumor invasion and metastasis [7-9]. Studies have reported that AS events occur more frequently in cancer tissues than in normal tissues, and changes can occur in both exons and introns [1012]. SF genes act as transcription factors to recognize the cis-regulatory element in pre-mRNA, then promote exon selection and splicing site selection [13]. SFs play important role in tumors by affect AS events in a variety of ways [14].

$\mathrm{OC}$ is the most fatal disease among gynecological malignancies. It is the 10th leading cause of cancer death among women in China and the fifth leading cause of cancer death among women globally. According to statistics, the five-year survival rate of 2010-2016 in OC was $48.6 \%$ (https://seer.cancer.gov/statfacts/html/ ovary.html) .In 2018, there were 295,414 OC patients in the world, among which 52,971 were in China. The late diagnosis and the occurrence of platinum-resistance have great impact on the prognosis of patients. It is important to discover high sensitive and specific biomarkers for OC patients. Previous studies have shown that there are close association between AS events and $\mathrm{OC}$, but the systematic analysis of them on the prognosis is still not comprehensive [15]. Similarly, the relationship between splicing factor and $\mathrm{OC}$ is less about prognosis.

Bioinformatics technology can use biological algorithms and related software tools to collect, process, store, analyze and interpret biological data. High-throughput sequencing technology is a relatively mature sequencing technology in recent years, which makes it possible to analyze the whole transcriptome and genome of species. In the study of AS events, RNA sequencing technology(RNA-seq) of high-throughput sequencing technology was mostly adopted, and full transcriptome sequencing was performed at the transcriptome level. Studies have shown that RNA-seq is also very good at detecting those genes closely related to $\mathrm{OC}[16,17]$. In this study, a large number of OC raw data were obtained from the TCGA database, and a systematic analysis of AS events and the prognosis of OC patients was conducted from aspects such AS image and signal processing by using bioinformatics software combined with RNA-seq. We found that a large number of AS events were related to the OS of OC and SFs, and the constructed prognostic models were of good value in evaluating the prognosis of patients.

\section{Material and methods \\ Data acquisition and preprocessing}

The clinical and transcriptomic data of 587 OC samples were downloaded from TCGA database (https://tcgadata.nci.nih.gov/tcga/). The sample ID was converted to the form of "OSCAR|51769|AA" (OSCAR is gene symbol, 51769 is ID number and AA is splicing type), samples with unquantifiable values greater than $30 \%$ were deleted. The Percent- splice-in (PSI) value of AS events were download from the TCGA SpliceSeq database (https://bioinforma tics.mdanderson.org/TCGASpliceSeq/PSIdownload.jsp), which included seven types AS events with PSI values: Exon skip (ES), both intron (RI), Alternate Donor site (AD), Alternate acceptor site (AA), Alternate promoter (AP), Alternate terminator (AT), and Channel exclusive exons (ME). The PSI values ranged from $0 \%$ to $100 \%$, the data with mean PSI $<0.05$, fluctuation $<0.01$, NA probability $>30 \%$ and unknown survival state were deleted . Finally, 384 samples with survival time more than 90 days were included in the study. Through other articles [18], we obtained 404 human SF genes for this study.

\section{Getting the survival-associated AS events}

Univariate Cox regression analysis was used to obtain OS-related AS events in R4.0.3. The relationship between AS events and prognosis of OC was understood by drawing volcano plot. Volcano Plot based on the correspondence between $-\log 10$ and $\mathrm{Z}$ value, red represents the AS events with $P<0.05$, and blue represents the AS events with $P>0.05$. The comparison of seven types AS events were made in Upset plot. Histogram shows the high and low risk group in each type according to the relationship between $H R$ value and the prognosis.In the bubble diagram, the correlation between AS events and prognosis was indicated by color and circular size.

\section{Building and evaluating survival model}

The optimal number of AS events were obtained by Least absolute shrinkage and selection operator (LASSO) regression. The screened AS events were analyzed by $\mathrm{R}$ package and the survival model formula was constructed as follows:Riskscore $=\sum_{i}^{n}$ PSI $\beta \mathrm{i}$ ( $\beta$ is the regression coefficien $_{t}$ ) [5]. After calculating the median value of the risk score, we divided the samples into high risk group and low risk group. Kaplan-Meier survival curves were 

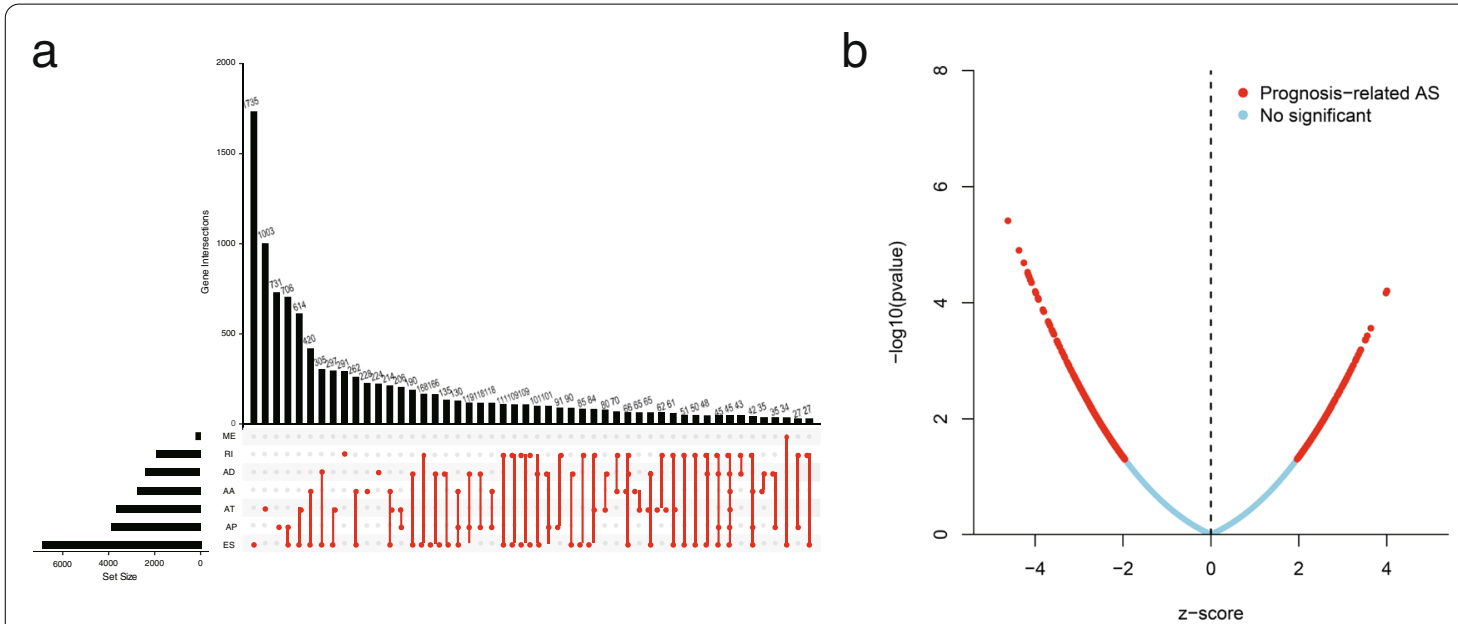

C

d
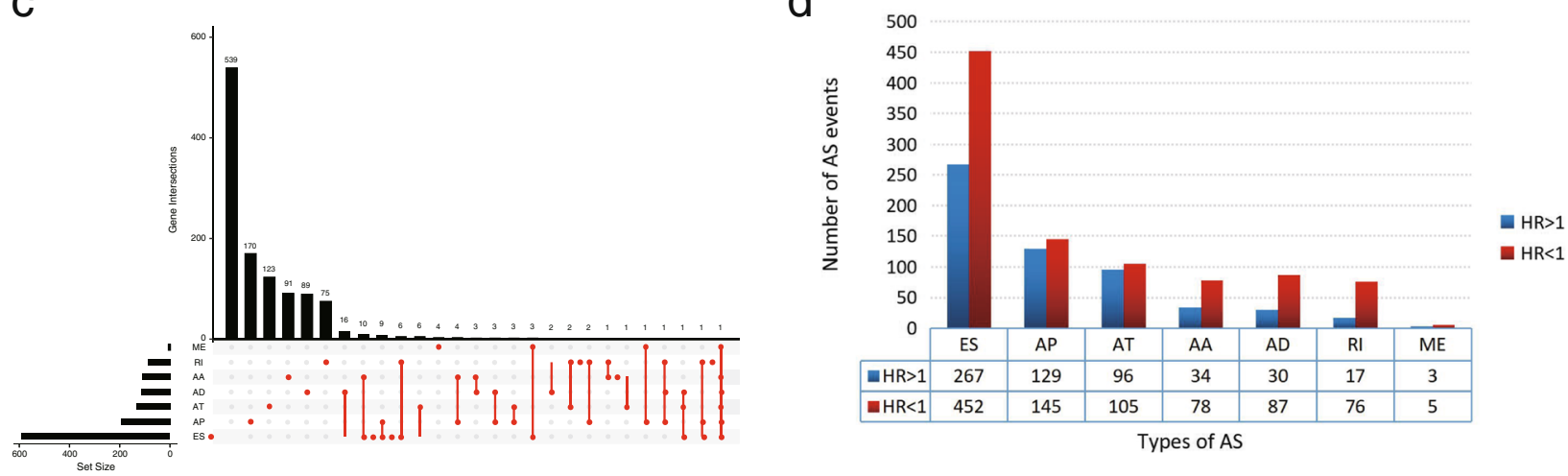

Fig. 1 Total analysis of alternative splicing events and ovarian cancer. a, An Upset plot displaying the intersections of different types of alternative splicing events associated with survival in ovarian cancer in ovarian cancer. $\mathbf{b}$, Volcanic Plot showing the strength of association between alternative splicing events and ovarian cancer. c, An Upset plot displaying the intersections of different types of alternative splicing events significantly associated with survival in ovarian cancer in ovarian cancer $(P<0.05)$. d, Among alternative splicing events significantly associated with ovarian cancer survival, the number of positive $(H R<1)$ or negative $(H R>1)$ events. AA, alternate acceptor site; $A D$, alternate donor site; $A P$, alternate promoter; AT, alternate terminator; ES, exon skip; ME, mutually exclusive exons; RI, retained intron

drawn and the difference of the two groups were represented by the $P$ value [19]. In addition, the relationship between the risk score and prognosis in OC patients were analyzed by risk score curves, survival status diagrams and PSI value heatmap jointly. The accuracy of survival models was evaluated by ROC. Then, single and multifactor independent prognostic analysis were carried out to evaluate the independent factors related to the prognosis of OC patients.

\section{Constructing correlation network between SF genes and AS events}

The date that absolute values of correlation coefficient $>0.4$ ( $\mathrm{Z}$ value) and $P$ value $<0.001$ were screened, then the network map between SF genes and AS events was constructed in Cytoscape to demonstrate the regulatory role of SF gene and AS events in OC. Joint use of DAVID database (https://david.ncifcrf.gov/) and KOBAS database (http://kobas.cbi.pku.edu.cn/kobas3) to Gene Ontology(GO) and Kyoto Encyclopedia of Genes and Genomes(KEGG) analysis for the 109 SFs. Similarly, R was used to visualize the KEGG analysis results and the top $20 \mathrm{GO}$ analysis pathways with the smallest $P$ values.

\section{Results}

\section{Different types of AS events in OC}

A total of 10,582 genes had 48,049 events occur in OC.There were seven AS events types in OC,among which ESs is the most common, with 19,251 occurring in 6931 genes, followed by APs and ATs corresponding to 9,689 and 8,453 AS events. The least number was 207 MEs occurring in 201 genes, and the rest were 4006 AAs and 3,497 ADs (Fig. 1a). 


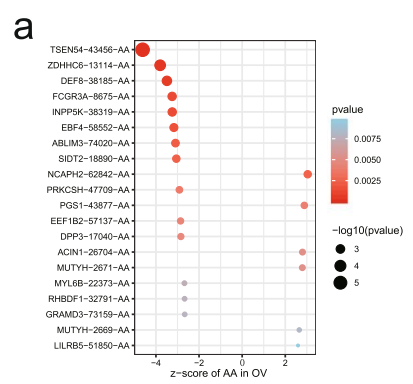

e

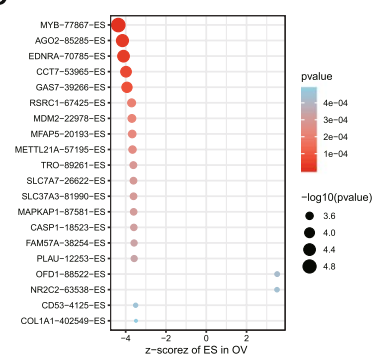

b

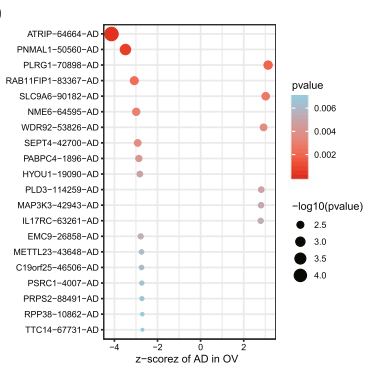

f

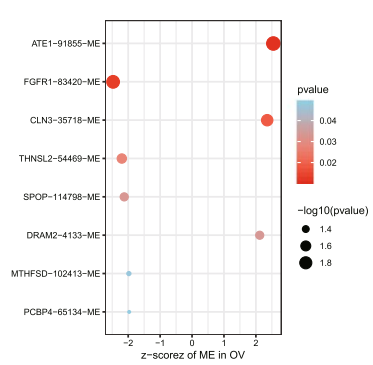

C

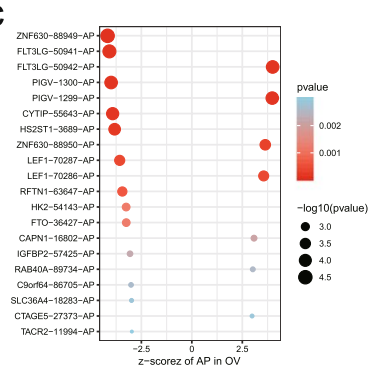

g

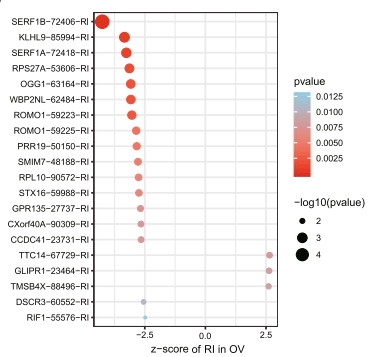

d
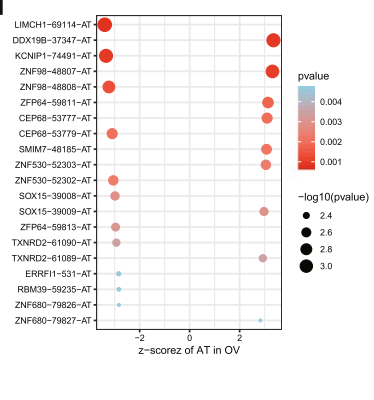

Fig. 2 Bubble plots of the alternative splicing events significantly associated with survival in ovarian. The red color and size of the bubble were positively correlated with the survival correlation. There is a corresponding relationship between Z-score and P value in the plots. AA, alternate acceptor site; AD, alternate donor site; AP, alternate promoter; AT, alternate terminator; ES, exon skip; ME, mutually exclusive exons; RI, retained intron

\section{OS-related AS events in OC patients}

Univariate COX regression analysis of 384 samples showed that there were 31,286 OS related AS events (Additional file 1: Table 1). Volcanic Plot visualizes these AS events (Fig. 1b).Upset plot shows that a gene can have one to seven types of AS events (Fig. 1c).After screening,1524 AS events were significantly correlated with prognosis (Additional file 2: Table 2). In the histogram (Fig. 1d),there are more AS events in each splicing type are conducive to survival than those are bad for survival. The top 20 AS events ( 8 of ME) in each splicing type are represented by the bubble plots (Fig. 2).

\section{Establishing OC survival model}

The lambda were selected by Lasso regression (Fig. 3). The adjusted lambda had 17, 20, 14, 20, 14, 8, 16,19AS events corresponding to AP, AA, AT, AD, ES, ME, RI and total splicing events, respectively Fig. 4.

The Multivariate Cox regression model established eight Cox proportional hazards regression prediction models (Additional files 3, 4, 5, 6, 7, 8, 9, 10, $11,12,13,14,15$ and 16: Tables $3,4,5,6,7,8,9,10$, $11,12,13,14,15$ and 16). The relationship between risk values and prognosis in the high and low risk group was represented by risk score curves,survival status and survival timesplots and PSI value heatmaps (Figs. 5, 6, and 7).The survival curve compared the survival differences between the high-low risk groups. Among the eight models, the difference in
AP was the largest and the difference in ME was the smallest (Fig. 8).

ROC curve evaluated 8 prognostic models, the AUC were between 0.680-0.757 (Fig. 9). The AUC of ME and AT were the largest and equal, while the area under the curve of RI and ES were less than 0.70.The evaluation results indicated that the prognostic modes were of great significance in the analysis of the prognosis of $\mathrm{OC}$ patients.

\section{Independent prognostic analysis of $O C$ patients}

Both single-factor independent prognostic analysis and multivariate independent prognostic analysis showed that age and risk score could be used as independent risk factors affecting OS (Fig. 10). In the single-factor independent prognostic analysis (Additional file 17: Table 17), the analysis results of age were $\mathrm{P}=$ $0.015, \mathrm{HR}=1.016$, and 95\%HR CI[1.003-1.030], and the analysis results of risk score were $\mathrm{P}<0.001, \mathrm{HR}$ $=1.049$, and 95\%HR CI[1.034-1.0640].In the multivariate independent prognostic analysis(Additional file 18: Table 18), the analysis results of age were $\mathrm{P}=0.023, \mathrm{HR}=1.015,95 \% \mathrm{HR} \mathrm{CI}[1.002-1.029])$ and the analysis results of risk score were $\mathrm{P}=0.023, \mathrm{HR}=1.015$, 95\%HR CI[1.002-1.029].

\section{Correlation analysis of AS events and SFs in OC}

Univariate COX regression analyzed the correlation between SF genes and AS events.The results showed 

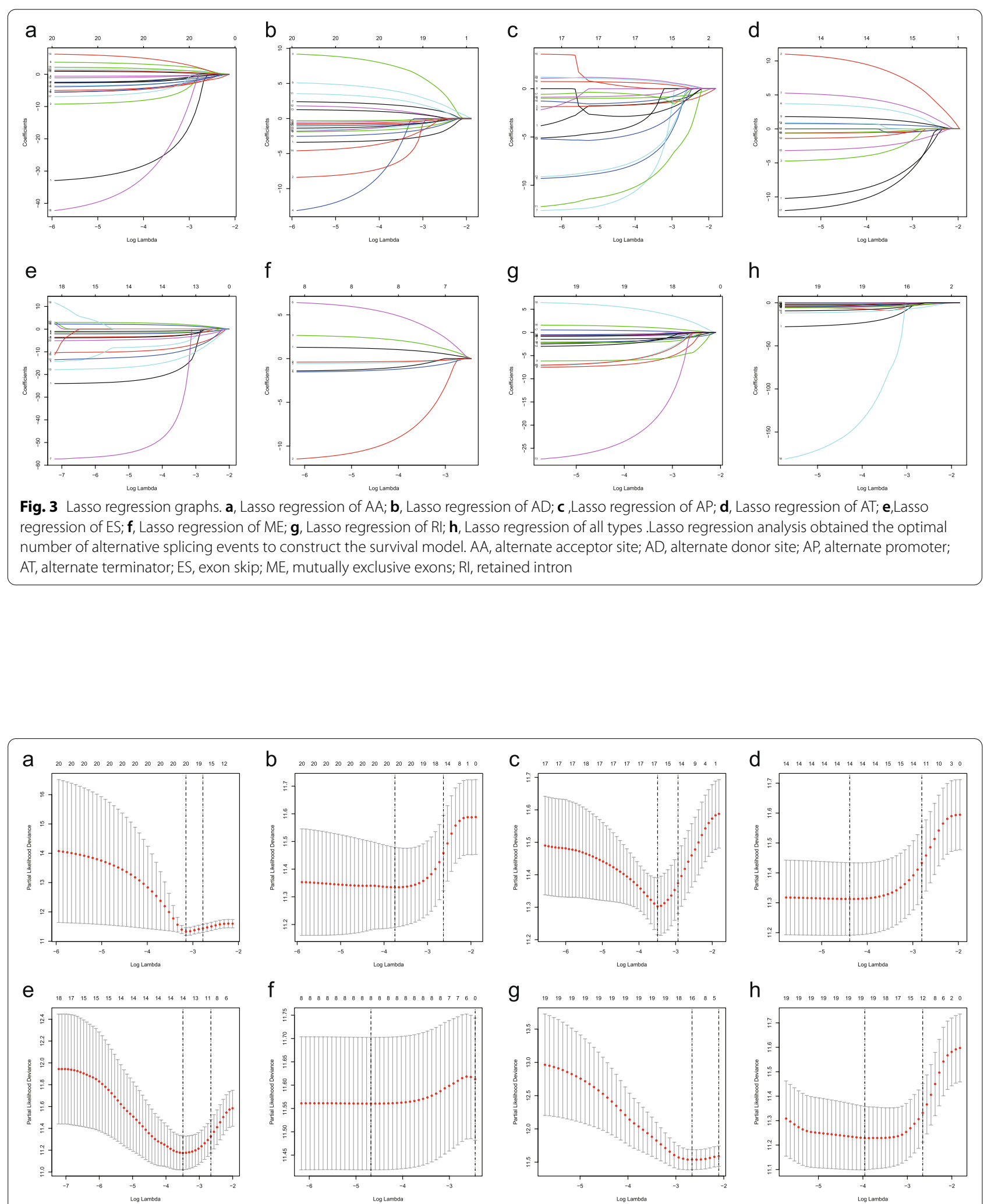

Fig. 4 Lasso regression graphs.a,Lasso regression of AA; $\mathbf{b}$,Lasso regression of $A D ; \mathbf{c}$, Lasso regression of AP; $\mathbf{d}$,Lasso regression of AT;e, Lasso regression of $E S ; \mathbf{f}$, Lasso regression of $\mathrm{ME} ; \mathbf{g}$, Lasso regression of $\mathrm{Rl} ; \mathbf{h}$, Lasso regression of all types.Lasso regression was used to obtain the data with the minimum error of the survival model.AA, alternate acceptor site; AD,alternate donor site; AP, alternate promoter; $A T$, alternate terminator;ES, exon skip; ME, mutually exclusive exons; Rl, retained intron 


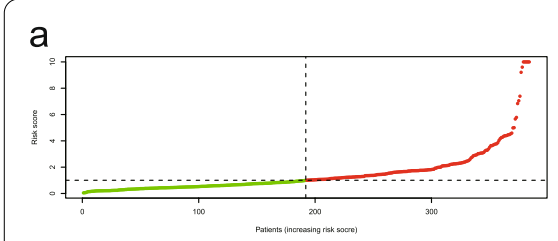

d

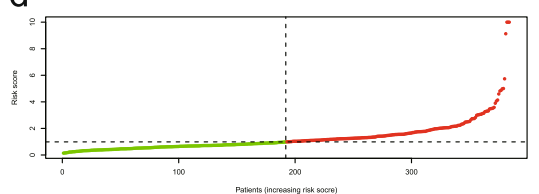

$\mathrm{g}$

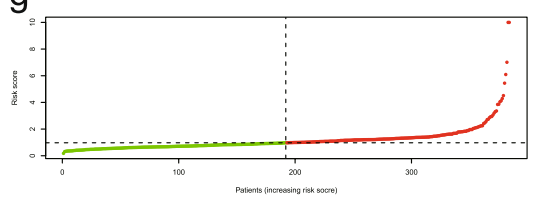

b

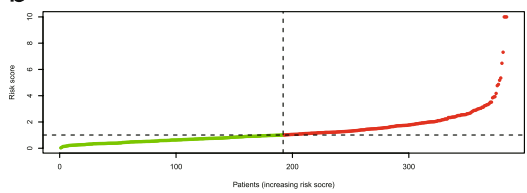

e

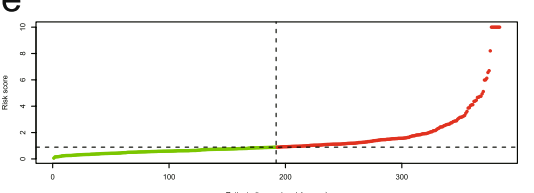

$\mathrm{h}$

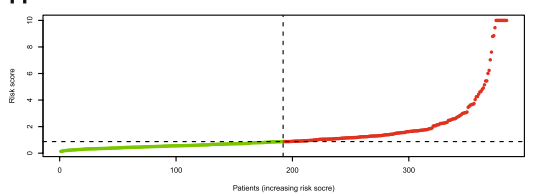

C

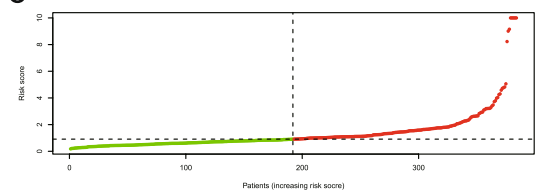

f

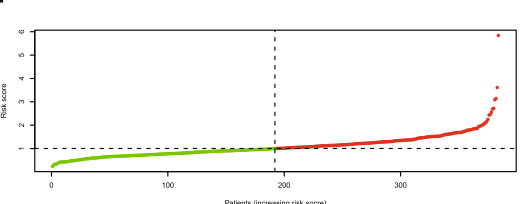

Fig. 5 Risk curves of different splicing types in ovarian cancer. $\mathbf{a}$, Risk curve of AA; $\mathbf{b}$, Risk curve of AD; $\mathbf{c}$, Risk curve of AP; $\mathbf{d}$, Risk curve of AT; e, Risk curve of ES; $\mathbf{f}$, Risk curve of ME; $\mathbf{g}$, Risk curve of Rl; $\mathbf{h}$, Risk curve of all types. Green means low risk, red means high risk. AA, alternate acceptor site; AD, alternate donor site; AP, alternate promoter; AT, alternate terminator; ES, exon skip; ME, mutually exclusive exons; RI, retained intron
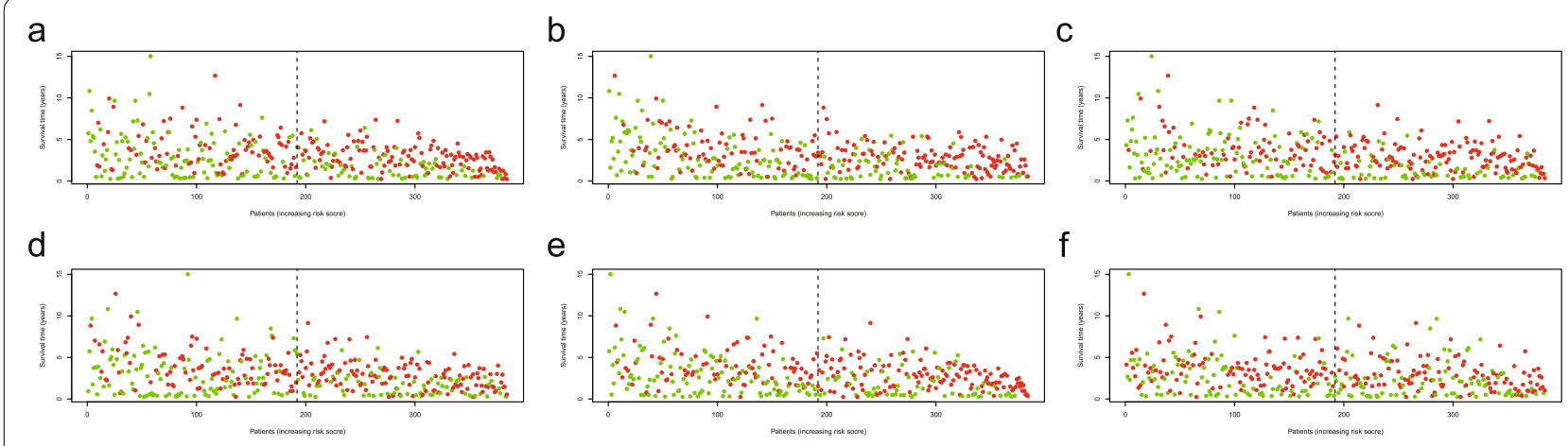

e

f
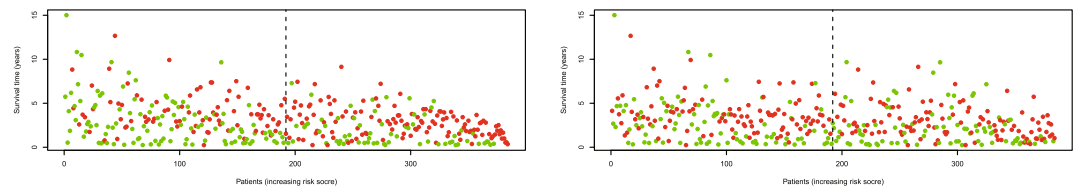

g

$\mathrm{h}$
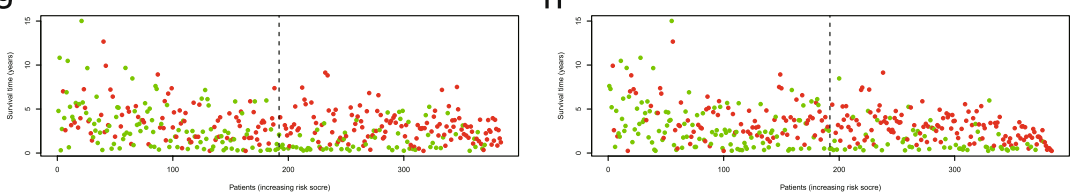

Fig. 6 Survival state diagram of ovarian cancer. a, Survival state diagram of AA; $\mathbf{b}$, Survival state diagram of AD; $\mathbf{c}$, Survival state diagram of AP; $\mathbf{d}$, Survival state diagram of AT; $\mathbf{e}$, Survival state diagrame of ES; $\mathbf{f}$, Survival state diagram of ME; $\mathbf{g}$, Survival state diagram of Rl; $\mathbf{h}$, Survival state diagram of all types. The abscissa survival time lengthened gradually, and the green dots represent survival, the red dots represent death. AA, alternate acceptor site; $A D$, alternate donor site; $A$, alternate promoter; $A T$, alternate terminator; $E S$, exon skip; $M E$, mutually exclusive exons; $R$, retained intron

that 109 SFs were associated with 324 AS events (Additional file 19: Table 19).In the network constructed by Cytoscape (Fig. 11), the relationship between SF genes and AS events are not only one-to-one, but many-toone or many-to-many.In addition, SFs have positive and negative adjustments for high and low risk events. As shown in the figure, the partial results of GO and KEGG enrichment analysis showed that 109 SFs were involved in a variety of biological processes such as RNA transport, degradation, processing and so on (Fig. 12).

\section{Discussion}

$\mathrm{OC}$ is one of the diseases with poor prognosis in gynecological malignancies. In recent years, finding effective prognostic biomarkers for $\mathrm{OC}$ has become a hot spot, and 


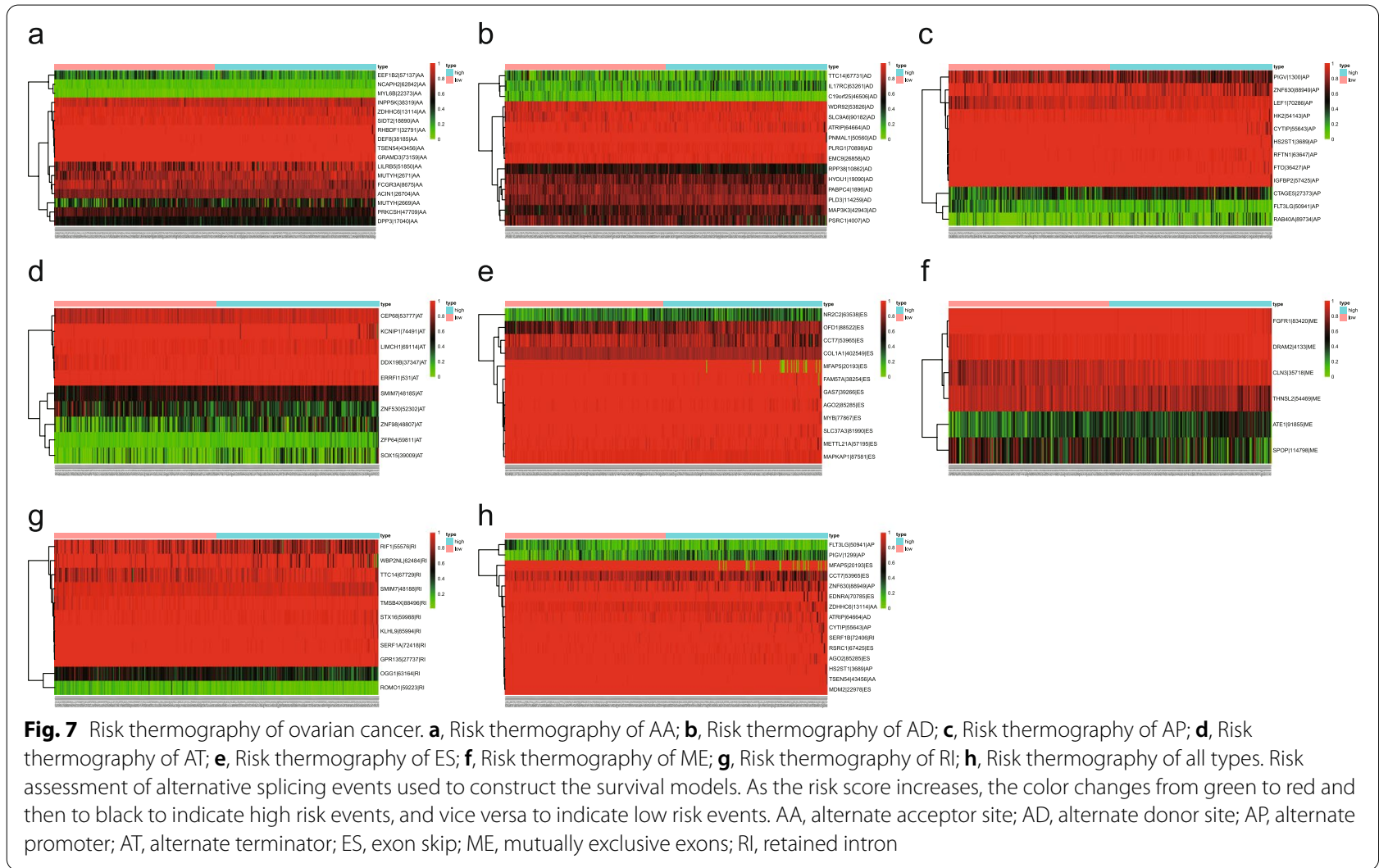

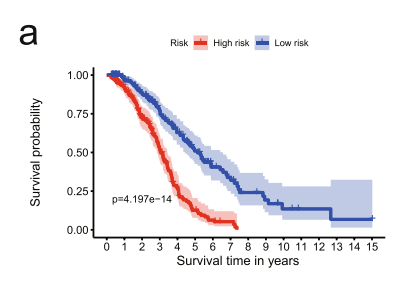

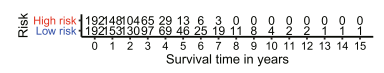

e

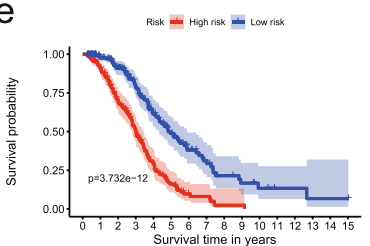

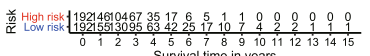

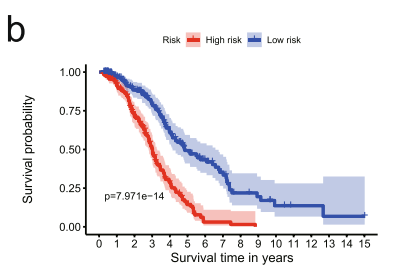

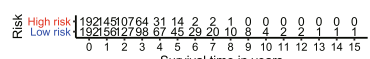

f

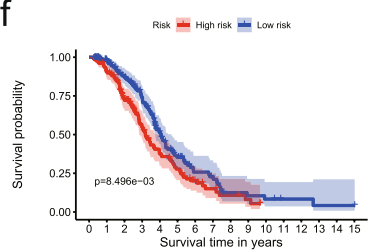

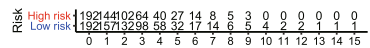

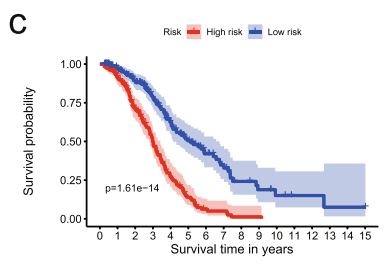

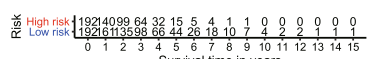

g

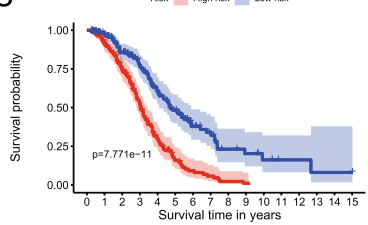

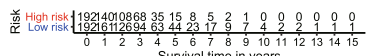

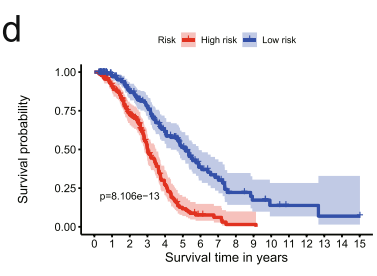

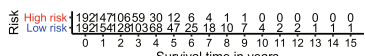

$\mathrm{h}$

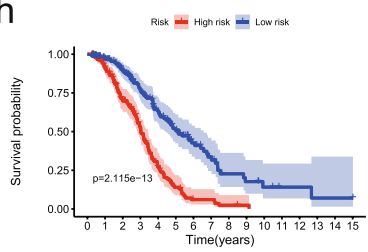

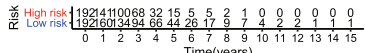

Fig. 8 Survival curves of ovarian cancer. a, Survival curve of AA; b, Survival curve of AD; $\mathbf{c}$, Survival curve of AP; d, Survival curve of AT; e, Survival curve of ES; $\mathbf{f}$, Survival curve of ME; $\mathbf{g}$, Survival curve of Rl; $\mathbf{h}$, Survival curve of all types. The survival curves show the differences in survival between the high and low risk groups. AA, alternate acceptor site; $A D$, alternate donor site; $A$, alternate promoter; $A T$, alternate terminator; ES, exon skip; ME, mutually exclusive exons; Rl, retained intron 


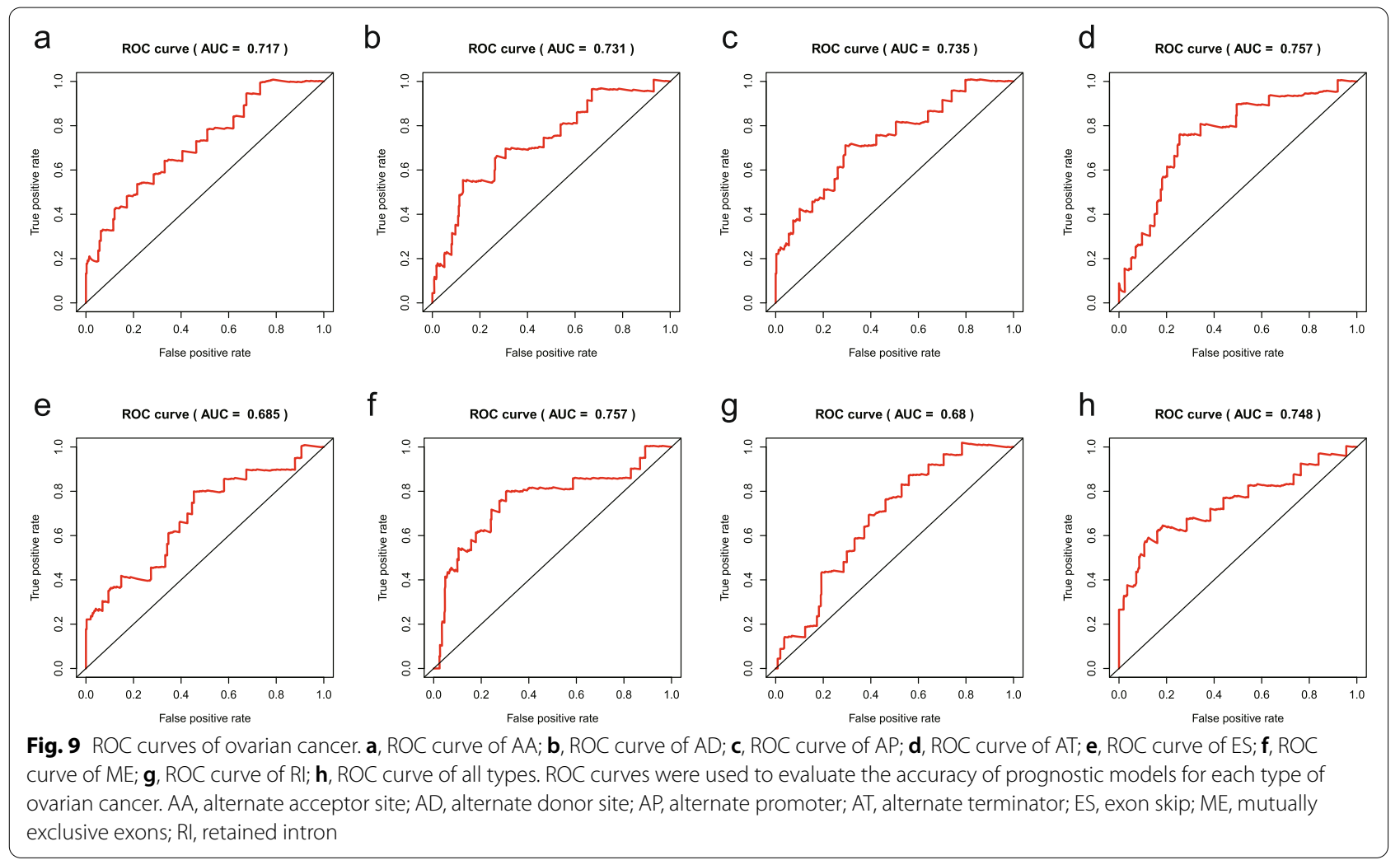

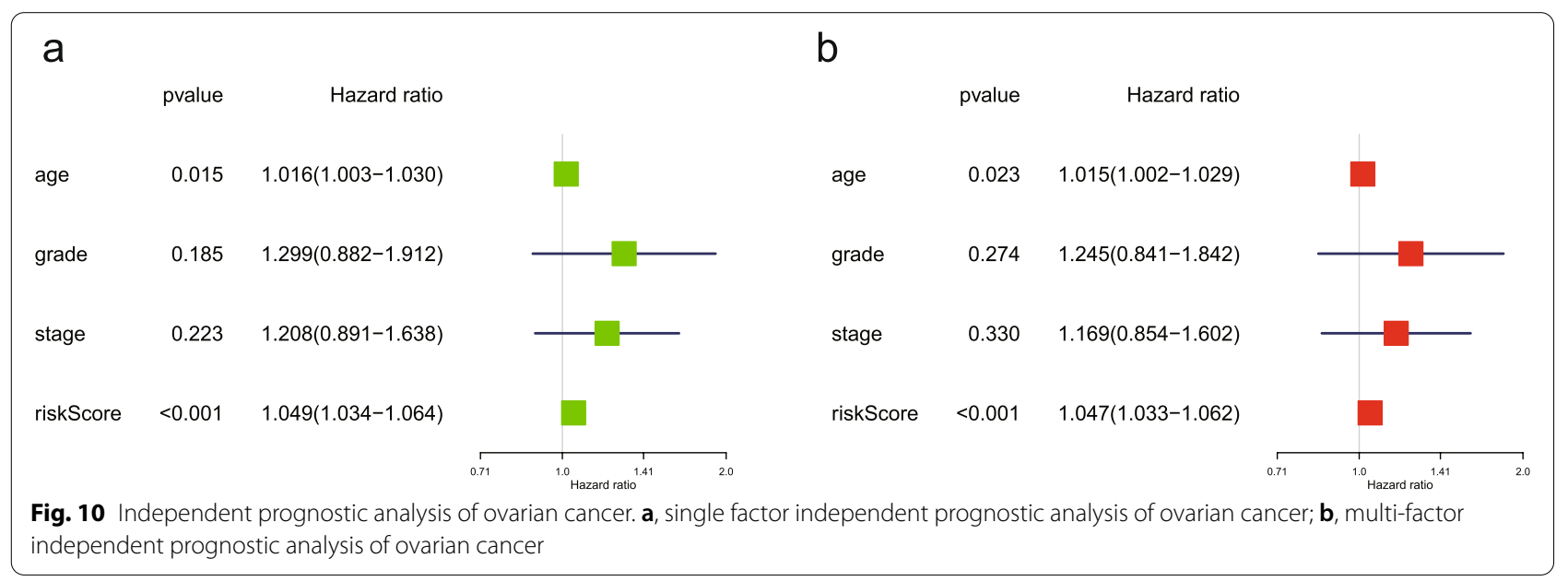

it is also an urgent problem to improve the prognosis of OC patients.As is one of the important processes in the regulation of protein diversification. Abnormal regulation of AS can affect human health and also play a role in some cancers.Previous reports have explored the relationship between AS and OC. In this report,the features of the relationship among prognosis, AS and SFs of OC were explored in a more comprehensive way.Besides,some independent prognostic factors of $\mathrm{OC}$ were analyzed, providing important basis for systematic analysis.

Prognostic exploration of OC mainly focuses on genomics, in addition to proteomics, immunology, cytokines and other aspects, among which there are complex relationships.For example, the tumor suppressor genes BRCA1 and BRCA2, whose mutations undergo a large number of splicing events, are important components of inherited breast and OC [20-22]. Further 


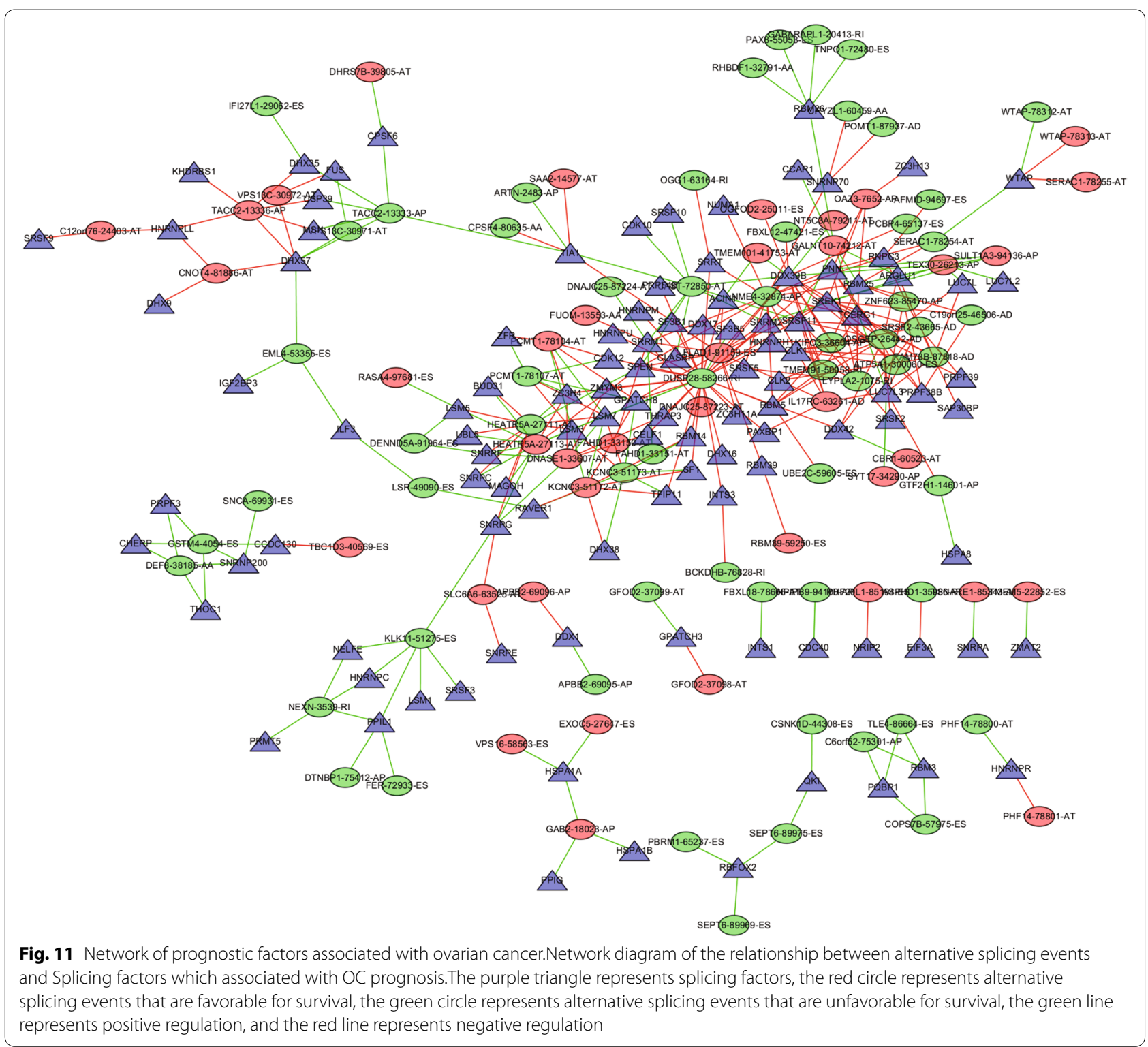

investigation revealed that the elimination of BRCA1 mutations through selective mRNA splicing would benefit patients resistant to OC [23]. Recent studies have shown that AAT, NFkB, PMVK and some other proteins are significantly higher in high grade serous ovarian carcinoma than in normal tissue, which can be used as biomarkers for predicting good prognosis [24].Correspondingly, such as PD-1, PD-L1, VAP1, FABP4, and PF4 are associated with poor prognosis [25]; . Interleukin is a series of cytokines produced by many kinds of cells and used a series of cells. There are 38 kinds of interleukin in this family, which can regulate a variety of immune processes.Among them, IL-17 [26, 27], IL-6 [28], IL-8 [28], IL-33 [27], CYR61 [29] and some other cytokines may be involved in the occurrence and development of $\mathrm{OC}$ and may be used as prognostic markers of OC.

In this study, we used data from TCGA database, combined with high-throughput sequencing technology and bioinformatics to analyze the relationship between AS, SF and the prognosis of OC.In addition, independent prognostic factors of OC, GO, KEGG analysis of pathways for prognostic related SFs were also explored.Consistent with other studies, our study shows that AS events and SFs play a role in $\mathrm{OC}$, and many splicing events are associated with OC.With the increase of the sample size in this study, the number of AS events in OC was also 48049, with the highest number of ES type [15]. After further treatment, the AS events of TSEN54, MYB, SERF1B, ZNF630 and 

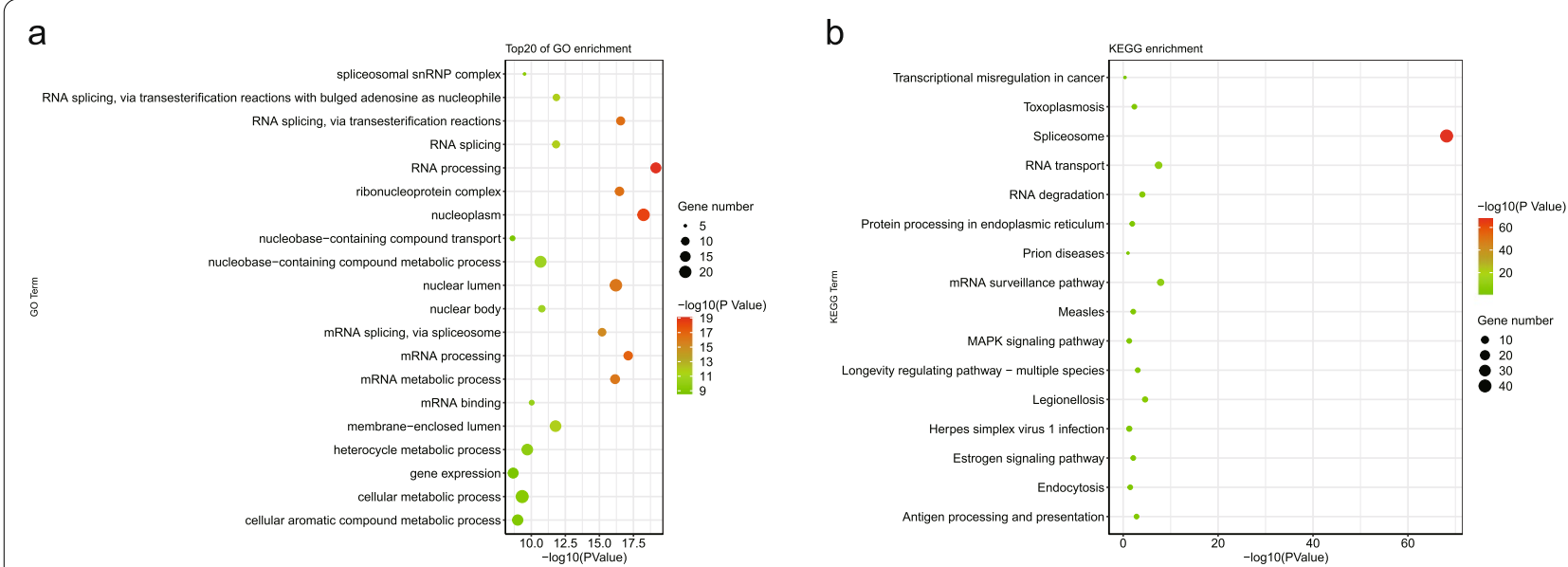

Fig. 12 Enrichment analysis of splicing factors. a, Gene Ontology enrichment analysis of splicing factors; $\mathbf{b}$, Kyoto Encyclopedia of Genes and Genomes enrichment analysis of splicing factors

AGO2 were most closely associated with the prognosis of $\mathrm{OC}$, and they were all good prognostic factors.tRNA splicing endonuclease(TSEN) catalyz tRNA intron removal in eukaryotes,and TSEN54 is a TSEN is one of the core subunits.The gene mutation of TSEN54 mostly leads to nervous system lesions, most studied cause of pontocerebellar hypoplasia.There has been a lack of research on the relationship between OC [30-32]. Previously,MYB is one of the proto-oncogenes, it has become a consensus that there is a relationship between MYB overexpression and poor prognosis of OC. Using the correlation between MYB and $\mathrm{OC}$ to evaluate the prognosis and explore potential therapeutic targets has attracted much attention [33-35]. But, the study found that MYB-77867-ES for OC prognosis is favorable AS event. The results suggest that splicing may change the role of genes.AGO2 is a Protein Coding gene, which have effect on cervical cancer, breast cancer and other tumors [36-38]. AGO2 fail in our study it happened happened AGO2-85285-ES is beneficial to the prognosis of OC, but in other studies, AGO2 can have opposite effects on the prognosis of $\mathrm{OC}$ through different pathways [39-41]. Few studies have been done on SERF1B and ZNF630,we get SERF1B-72406-RI $、$ ZNF630-88949-AP close contact with $\mathrm{OC}$, they may be effective prognostic biomarkers and therapeutic targets.Among the remaining results, there are many previous studies on CD44 gene.As a non-kinase transmembrane receptor,CD44 can be used as an effective biomarker to predict the prognosis of $\mathrm{OC}$ and negatively affect the outcome of OC [42]. CD44 plays a multi-functional role, such as related to cancer stem cells and tumor-associated macrophages, leading to drug resistance of recurrent chemotherapy drugs in OC. It is expected to further dig out effective therapeutic targets to reduce the recurrence rate and drug resistance rate of
OC [43, 44]. It interacts with STAT3 to affect a series of processes such as angiogenesis and immune regulation in OC [45-47]. CD44 and STAT3 work together on OC in a variety of way,which can provide various ideas for $\mathrm{OC}$ treatment. Consistent with previous researchs, we get CD44-15112-ES can negatively affect the prognosis of OC.

Our carefully designed analysis scheme, it lays the foundation for the feasibility of the prediction models.LASSO regression has unique advantages for survival analysis, which can reduce the complexity of prognostic features, select good model data, and improve the feasibility and accuracy of subsequent survival models [48]. We made full use of the advantages of LASSO regression model in Cox model to further optimize the construction of previous survival models. The survival model was evaluated by ROC curve, and the AUC of the eight models was between 0.68 and 0.757 , all of which had good accuracy and feasibility.

Independent prognostic factors are often used as a part of prognostic monitoring and usually divided into clinical features and molecular biology.Common clinical features such as age, performance status, grade, Figo stage, and histology were considered independent prognostic factors for OC $[49,50]$. On the other hand, researchers found that low level of AK7, high expression of KIF23, P-gp protein, and SIX-gene signature(TGFBI, SFRP1, COL16A1, THY1, PPIB, BGN) could all be considered independent prognostic factors for OC [51-54]. In this study, we analyzed some clinical traits and found that age can be used as an independent prognostic factor for OC.However, grade and stage were not significant as independent prognostic factors of $\mathrm{OC}$ in our study,this may be due to differences in research data and experimental methods.At the same time, we added the score of risk calculated by the prognostic model 
for evaluation, and risk score was also an independent prognostic factor. This result further demonstrates the strong applicability of the prognostic models, and this design method is relatively new in independent prognostic analysis.

The influence of AS on cancer is beyond question. SF gene indirectly affects the occurrence and development of cancer by affecting AS process $[14,55]$. There have been studies on the prognostic relationship between SFs and OC, and the SF we studied came from the data obtained after comprehensive analysis. More SF numbers may lead to more prognostic markers [15]. In this study, 109 SF genes were found to be most relevant for OC prognosis, among which MSI1 is an RNA binding protein that regulates the expression of target mRNAs at the translation level.Initial studies have shown that miR-761 reduces the proliferation and aggressiveness of OC by regulating MSI1 [56]. The increased expression of MSI1 can enhance the malignant characteristics of OC.In addition, MSI1 can increase the expression of p-Bcl-2 by activating the ERK signaling pathway and indirectly increase the drug resistance of chemotherapy drugs [57]. We found that MSI1 can produce positive and negative regulation on different AS events. Incidentally, MSI1 produces positive regulation on negative AS events, while produces negative regulation on positive AS events, and ultimately has negative effects on OC outcomes.RBM3 is the members of the cold shock protein family, it has been shown in a series of studies to be related to the prognosis of breast cancer [58], stomach cancer [59], OC [60], colorectal cancer [61], prostate cancer [62] and other malignant tumors..Asa Ehlen and his team suggest that RBM3 may be an independent prognostic factor for $\mathrm{OC}$, increasing the sensitivity of patients to chemotherapeutic agents and extending the survival of OC patients [63]. Inhibition of Protein Coding gene DDX39B high expression can improve the efficacy of chemotherapy drugs for OC. This is the only study between OC and DDX38B [64]. We found that RBM3 as SF negatively regulates the COPS7B-57975 -ES, and COPS7B- 57975-ES is a good prognostic factors.In other words, RBM3 has a negative prognostic effect on OC.Similarly, the results of this study showed that DDX39b positively affected the prognosis of OC by affecting AS events. This difference may be caused by the mechanism involved in RBM3 and DDX39b that has not been found yet, which needs to be further explored. Among the remaining SFs, the relationship between SFs such as PPIL1, SNRNP200, PQBP1, GPATCH8 and OC have not been explored yet, which can be further studied in the future.

In this paper, the relationship between the prognosis of OC,AS, SF were further explored, but there are still
shortcomings.First, the study lacked a control cohort due to the lack of adjacent normal samples around the tumor tissue.Second, the data analyzed in this study came from TCGA and relevant literature, all of which were online data, and the results may not be applicable to clinical patients.Third, the current lack of network data research results applicable to clinical OC patients, the urgent need to establish a clinical cohort to verify the results.Finally, the mechanism of the pathway obtained by GO and KEGG enrichment analysis can be further excavated.

\section{Conclusions}

In conclusion, our results indicated that AS can affect the occurrence and development of $\mathrm{OC}$, and ultimately affect the prognosis of ovarian cancer.On the other hand, SFs can indirectly act on multiple processes of OC development by regulating AS events, and have impact on the survival of $\mathrm{OC}$ patients.Further experiments are needed to explore participating pathways to develop effective prognostic biomarkers.

\section{Abbreviations}

OC: Ovarian cancer; AS: Alternative splicing; ROC: Time-dependent receiver operating characteristic; SFs: Splicing factors; OS: Overall survival; AUC: Area under the curve; pre-mRNA: Precursor messenger RNA; HCC: Hepatocellular carcinoma; RNA-seq: RNA sequencing; AA: Alternate acceptor site; AD: Alternate donor site; AP: Alternate promoter; AT: Alternate terminator; ES: Exon skip; ME: Mutually exclusive exons; RI: Retained intronPSI:Percent- splice-in; LASSO: Least absolute shrinkage and selection operator; GO: Gene Ontology; KEGG: Kyoto Encyclopedia of Genes and Genomes.

\section{Supplementary Information}

The online version contains supplementary material available at https://doi. org/10.1186/s13048-021-00866-1.

Additional file 1:Table 1. Relationship between alternative splicing events and ovarian cancer after univariate Cox regression analysis. $H R$, hazard ratio, $H R>1$ are high-risk events, $H R<1$ are low-risk events. The 95\% confidence interval from HR.95L to HR.95H.

Additional file 2: Table 2. Alternative splicing events significantly associated with prognosis of ovarian cancer.The $P$ values of all the alternative splicing events were less than 0.05.HR,hazard ratio,HRHR>1 are high-risk events, $\mathrm{HR}<1$ are low-risk events. The 95\% confidence interval from HR.95L to $\mathrm{HR} .95 \mathrm{H}$

Additional file 3: Table 3. Prognostic model of AA events in ovarian cancer.coef is the risk factor.

Additional file 4: Table 4. Risk score of AA events in ovarian cancer patients.

Additional file 5: Table 5. Prognostic model of AD events in ovarian cancer.coef is the risk factor.

Additional file 6: Table 6. Risk score of AD events in ovarian cancer patients.

Additional file 7: Table 7. Prognostic model of AP events in ovarian cancer.coef is the risk factor.

Additional file 8: Table 8. Risk score of AP events in ovarian cancer patients. 
Additional file 9: Table 9. Prognostic model of AT events in ovarian cancer.coef is the risk factor.

Additional file 10:Table 10. Risk score of AT events in ovarian cancer patients.

Additional file 11: Table 11. Prognostic model of ES events in ovarian cancer.coef is the risk factor.

Additional file 12: Table 12. Risk score of ES events in ovarian cancer patients.

Additional file 13: Table 13. Prognostic model of ME events in ovarian cancer.coef is the risk factor.

Additional file 14: Table 14. Risk score of ME events in ovarian cancer patients.

Additional file 15: Table 15. Prognostic model of Rl events in ovarian cancer.coef is the risk factor.

Additional file 16: Table 16. Risk score of Rl events in ovarian cancer patients.

Additional file 17: Table 17. Single factor independent analysis of ovarian cancer.The $95 \%$ confidence interval from HR.95L to HR.95H.P value less than 0.05 indicates that this factor is significant as an independent prognostic factor for ovarian cancer.

Additional file 18: Table 18. Multi-factor independent prognostic analysis of ovarian cancer.The $95 \%$ confidence interval from HR.95L to HR.95H.P value less than 0.05 indicates that this factor is significant as an independent prognostic factor for ovarian cancer.

Additional file 19: Table 19. Analysis of the relationship between splicing factors and variable splicing events.

Additional file 20: Table 20. Network node property sheet.

Additional file 21: Table 21. Results of splicing factors Gene Ontology enrichment analysis.Input number:The number of splicing factors involved in the pathway.Background number:Number of all genes involved in the pathway.

Additional file 22: Table 22. Results of splicing factors Kyoto Encyclopedia of Genes and Genomes enrichment analysis.Input number:The number of splicing factors involved in the pathway.Background number:Number of all genes involved in the pathway.

\section{Acknowledgements}

None.

\section{Authors' contributions}

DZ: Data collection, Data analysis, Manuscript writing. DZ: Data analysis and data collection. YD: Data analysis and data collection. LY: Project development and Manuscript writing. All authors read and approved the final manuscript.

\section{Funding}

None.

\section{Availability of data and materials}

Please contact the corresponding author Lihua Yang (13,759,481,789@163.com).

\section{Declarations}

\section{Consent for publication}

Not applicable.

\section{Competing interests}

None.

Received: 2 May 2021 Accepted: 30 June 2021

Published online: 15 September 2021

\section{References}

1. Lee, Yeon, Rio,D. C.Mechanisms and Regulation of Alternative Pre-mRNA Splicing. Annu Rev Biochem.2015; 84: 291-323.

2. Eric.T, Wang, Rickard, Sandberg, Shujun, Luo, et al.Alternative isoform regulation in human tissue transcriptomes. Nature.2008; 456(7221): 470-476.

3. Kashima, T.J. L. Manley.A negative element in SMN2 exon 7 inhibits splicing in spinal muscular atrophy. Nature Genetics.2003; 34(4): 460-463.

4. Chen X, Zhao C, Guo B, Zhao Z, Fang Z. Systematic Profiling of Alternative mRNA Splicing Signature for Predicting Glioblastoma Prognosis. Front Oncol. 2019;9:928.

5. Lingpeng, Yang, Zifei, Zhang, Wentao,Wang.Systematic analysis and prediction model construction of alternative splicing events in hepatocellular carcinoma: a study on the basis of large-scale spliceseq data from The Cancer Genome Atlas. PeerJ.2019; 7: e8245.

6. Zuo Y, Zhang L, Tang W, Tang W. Identification of prognosis-related alternative splicing events in kidney renal clear cell carcinoma. J Cell Mol Med. 2019;23(11):7762-72.

7. Wang F, Fu X, Chen P, Wu P, Fan X, Li N, et al. SPSB1-mediated HnRNP A1 ubiquitylation regulates alternative splicing and cell migration in EGF signaling. Cell Res. 2017;27(4):540-58.

8. Alison, Tyson-Capper, Hannah,Gautrey.Regulation of Mcl-1 alternative splicing by hnRNP F, H1 and K in breast cancer cells. RNA Biology.2018; 15(12): 1448-1457.

9. Xie, R., X. Chen, Z. Chen, M. Huang, W. Dong, P. Gu, et al.Polypyrimidine tract binding protein 1 promotes lymphatic metastasis and proliferation of bladder cancer via alternative splicing of MEIS2 and PKM. Cancer Letters.2019; 449: 31-44.

10. Dvinge $H$, Bradley RK. Widespread intron retention diversifies most cancer transcriptomes. Genome Med. 2015;7(1):45.

11. Jung H, Lee D, Lee J, Park D, Kim YJ, Park WY, et al. Intron retention is a widespread mechanism of tumor-suppressor inactivation. Nat Genet. 2015;47(11):1242-8.

12. Miri, Danan-Gotthold, Regina, Golan-Gerstl, Eli, Eisenberg, et al.Identification of recurrent regulated alternative splicing events across human solid tumors. Nucleic Acids Research.2015; 43(10): 5130-5144.

13. Shen, S., Y. Wang, C. Wang, Y. N. Wu, Y. Xing.SURVIV for survival analysis of mRNA isoform variation. Nature Communications.2016; 7(1): 11548.

14. Sveen A, Kilpinen S, Ruusulehto A, Lothe RA, Skotheim RI. Aberrant RNA splicing in cancer; expression changes and driver mutations of splicing factor genes. Oncogene. 2015;35(19):2413-27.

15. Zhu J, Chen Z, Yong L. Systematic profiling of alternative splicing signature reveals prognostic predictor for ovarian cancer. Gynecol Oncol Int J. 2018;148(2):368-74

16. Kannan, K., C. Coarfa, K. Rajapakshe, S. M. Hawkins, M. M. Matzuk, A. Milosavljevic, et al.CDKN2D-WDFY2 Is a Cancer-Specific Fusion Gene Recurrent in High-Grade Serous Ovarian Carcinoma. PLOS Genetics.2014; 10(3): e1004216-

17. Earp MA, Raghavan R, Li Q, Dai J, Fridley BL. Characterization of fusion genes in common and rare epithelial ovarian cancer histologic subtypes. Oncotarget. 2017;8(29):46891-9.

18. Felau; Melpomeni Kasapi; Martin L. Ferguson; Carolyn M. Hutter; M. S. S. P. A. A. A. J. P. T. T. P. Z. P. G. S. S. J. C.-J. J. A. D. I.Somatic Mutational Landscape of Splicing Factor Genes and Their Functional Consequences across 33 Cancer Types. Cell Reports.2018; 23(1): 282-296.

19. Rich, J. T., J. G. Neely, R. C. Paniello, C. C. Voelker, B. Nussenbaum,E. W. Wang.A practical guide to understanding Kaplan-Meier curves. Otolaryngol Head Neck Surg.2010; 143(3): 331-336.

20. Sanz DJ, Acedo A, Infante M, Duran M, Perez-Cabornero L, Esteban-Cardenosa $E$, et al. A high proportion of DNA variants of BRCA1 and BRCA2 is associated with aberrant splicing in breast/ovarian cancer patients. Clin Cancer Res. 2010;16(6):1957-67.

21. Zhang $L$, Chen L, Bacares R, Ruggeri JM, Somar J, Kemel Y, et al. BRCA1 R71K missense mutation contributes to cancer predisposition by increasing alternative transcript levels. Breast Cancer Res Treat. 2011;130(3):1051-6.

22. Colombo M, Blok MJ, Whiley P, Santamariña M, Gutiérrez-Enríquez S, Romero A, et al. Comprehensive annotation of splice junctions supports pervasive alternative splicing at the BRCA1 locus: a report from the ENIGMA consortium. Hum Mol Genet. 2014;23(14):3666-80. 
23. Wang Y, Bernhardy AJ, Cruz C, Krais JJ, Nacson J, Nicolas E, et al. The BRCA1- $\triangle 1$ 1q Alternative Splice Isoform Bypasses Germline Mutations and Promotes Therapeutic Resistance to PARP Inhibition and Cisplatin. Can Res. 2016;76(9):2778-90.

24. Kim SI, Jung M, Dan K, Lee S, Lee M. Proteomic Discovery of Biomarkers to Predict Prognosis of High-Grade Serous Ovarian Carcinoma. Cancers. 2020;12(4):790

25. Ju C, Gao JC, Zhang PX, Zhang KN, Yang S, Kang TJ, et al. Expression and significance of PD-1 and PD-L1 in the specimens of epithelial ovarian cancer. Zhonghua Fu Chan Ke Za Zhi. 2020;55(8):529-34.

26. Aotsuka A, Matsumoto Y, Arimoto T, Kawata A, Fujii T. Interleukin-17 is associated with expression of programmed cell death 1 ligand 1 in ovarian carcinoma. Cancer Sci. 2019;110(10):3068-78.

27. Sekiya A, Suzuki S, Tanaka A, Hattori S, Kikkawa F. Interleukin33 expression in ovarian cancer and its possible suppression of peritoneal carcinomatosis. Int J Oncol. 2019;55(3):755-65.

28. Rodrigues ISS, Martins-Filho A, Micheli DC, Lima CA, Tavares-Murta BM, Murta EFC, et al. IL-6 and IL-8 as Prognostic Factors in Peritoneal Fluid of Ovarian Cancer. Immunol Invest. 2020;49(5):510-21.

29. Shi, J., R. Huo, N. Li, H. Li,W. Di.CYR61, a potential biomarker of tumor inflammatory response in epithelial ovarian cancer microenvironment of tumor progress. BMC Cancer.2019; 19(1): 1140.

30. Simonati, A., D. Cassandrini, D. Bazan,F. M. Santorelli.TSEN54 mutation in a child with pontocerebellar hypoplasia type 1. Acta Neuropathologica.2011; 121(5): 671-673.

31. Maraş-Genç, H., E. Uyur-Yalçın, R. Rosti, J. G. Gleeson,B. Kara.TSEN54 generelated pontocerebellar hypoplasia type 2 presenting with exaggerated startle response: report of two cases in a family. Turk J Pediatr.2015; 57(3): 286-289.

32. Kasinathan A, Sankhyan N, Dijk TV, Singh P, Singhi P. Clinico-radiological Profile of Children with Pontocerebellar Hypoplasia. J Pediatr Neurosci. 2020;15(2):94

33. Miree O, Srivastava SK, Singh S, Scalici JM, Carter JE, Finan MA, et al. Overexpression of MYB is associated with growth and malignant behavior of ovarian cancer cells. Gynecol Oncol. 2018;149:63-4

34. Tung CH, Kuo LW, Huang MF, Wu YY, Tsai YT, Wu JE, et al. MicroRNA150-5 p promotes cell motility by inhibiting c-Myb-mediated Slug suppression and is a prognostic biomarker for recurrent ovarian cancer. Oncogene. 2020;39(4):862-76.

35. Li P, Xin H, Lu L. Extracellular vesicle-encapsulated microRNA-424 exerts inhibitory function in ovarian cancer by targeting MYB. J Transl Med. 2021:19(1):4.

36. Guo, J., J. Lv, M. Liu,H. Tang.miR-346 Up-regulates Argonaute 2 (AGO2) Protein Expression to Augment the Activity of Other MicroRNAs (miRNAs) and Contributes to Cervical Cancer Cell Malignancy. J Biol Chem.2015; 290(51): 30342-30350

37. Tilley, A. M. C., C. M. Howard, S. Sridharan, B. Subramaniyan, N. R. Bearss, S. Alkhalili, et al.The CXCR4-Dependent LASP1-Ago2 Interaction in TripleNegative Breast Cancer. Cancers (Basel).2020; 12(9): 2455.

38. Unal, O., Y. Akkoc, M. Kocak, E. Nalbat, A. I. Dogan-Ekici, H. Yagci Acar, et al.Treatment of breast cancer with autophagy inhibitory microRNAs carried by AGO2-conjugated nanoparticles. J Nanobiotechnology.2020; 18(1): 65

39. Cubillos-Ruiz JR, Baird JR, Tesone AJ, Rutkowski MR, Scarlett UK, Camposeco-Jacobs AL, et al. Reprogramming tumor-associated dendritic cells in vivo using miRNA mimetics triggers protective immunity against ovarian cancer. Cancer Res. 2012;72(7):1683-93.

40. Vaksman O, Hetland TE, Trope CG, Reich R, Davidson B. Argonaute, Dicer, and Drosha are up-regulated along tumor progression in serous ovarian carcinoma. Hum Pathol. 2012:43(11):2062-9.

41. Liao CC, Ho MY, Liang SM, Liang CM. Autophagic degradation of SQSTM1 inhibits ovarian cancer motility by decreasing DICER1 and AGO2 to induce MIRLET7A-3P. Autophagy. 2018;14(12):2065-82.

42. Martincuks, A., P. C. Li, O. Zhao, C. Zhang, Y. J. Li, H. Yu, et al.CD44 in Ovarian Cancer Progression and Therapy Resistance-A Critical Role for STAT3. Front Oncol.2020; 10: 589601.

43. Alvero AB, Chen R, Fu HH, Montagna M, Schwartz PE, Rutherford T, et al. Molecular phenotyping of human ovarian cancer stem cells unravels the mechanisms for repair and chemoresistance. Cell Cycle. 2009;8(1):158-66.
44. Yin M, LiX, Tan S, Zhou HJ, Ji W, Bellone S, et al. Tumor-associated macrophages drive spheroid formation during early transcoelomic metastasis of ovarian cancer. J Clin Invest. 2016:126(11):4157-73.

45. Högerkorp, C. M., S. Bilke, T. Breslin, S. Ingvarsson,C. A. Borrebaeck.CD44stimulated human B cells express transcripts specifically involved in immunomodulation and inflammation as analyzed by DNA microarrays. Blood.2003; 101(6): 2307-2313.

46. Cao G, Savani RC, Fehrenbach M, Lyons C, Zhang L, Coukos G, et al. Involvement of endothelial CD44 during in vivo angiogenesis. Am J Pathol. 2006;169(1):325-36.

47. Panni RZ, Sanford DE, Belt BA, Mitchem JB, Worley LA, Goetz BD, et al. Tumor-induced STAT3 activation in monocytic myeloid-derived suppressor cells enhances stemness and mesenchymal properties in human pancreatic cancer. Cancer Immunol Immunother. 2014;63(5):513-28.

48. Kostareli E, Hielscher T, Zucknick M, Baboci L, Wichmann G, Holzinger D, et al. Gene promoter methylation signature predicts survival of head and neck squamous cell carcinoma patients. Epigenetics. 2016;11(1):61-73.

49. du Bois A, Reuss A, Pujade-Lauraine E, Harter P, Ray-Coquard I, Pfisterer J. Role of surgical outcome as prognostic factor in advanced epithelial ovarian cancer: a combined exploratory analysis of 3 prospectively randomized phase 3 multicenter trials: by the Arbeitsgemeinschaft Gynaekologische Onkologie Studiengruppe Ovarialkarzinom (AGO-OVAR) and the Groupe d'Investigateurs Nationaux Pour les Etudes des Cancers de I'Ovaire (GINECO). Cancer. 2009;115(6):1234-44.

50. Zhang Y, Luo G, Li M, Guo P, Xiao Y, Ji H, et al. Global patterns and trends in ovarian cancer incidence: age, period and birth cohort analysis. BMC Cancer. 2019:19(1):984.

51. Hu Y, Zheng M, Wang C, Wang S, Gou R, Liu O, et al. Identification of KIF23 as a prognostic signature for ovarian cancer based on large-scale sampling and clinical validation. Am J Transl Res. 2020;12(9):4955-76.

52. Ji R, LiY, He C, Zhu X, He A, Lu Y. Detection and analysis of multiple biomarkers in ovarian cancer: clinical significance in diagnosis, treatment, and prognosis evaluation. Gland Surg. 2020;9(6):2175-86.

53. Pan, X.,X. Ma.A Novel Six-Gene Signature for Prognosis Prediction in Ovarian Cancer. Front Genet.2020; 11: 1006

54. Zhang, X. Y., L. L. Zhou, Y. Jiao, Y. Q. Li, Y. N. Guan, Y. C. Zhao, et al.Adenylate kinase 7 is a prognostic indicator of overall survival in ovarian cancer. Medicine (Baltimore).2021; 100(1): e24134.

55. Dvinge, H., E. Kim, O. Abdel-Wahab,R. K. Bradley.RNA splicing factors as oncoproteins and tumour suppressors. Nature Reviews Cancer. 16(7): 413-430.

56. Shi, C.,Z. Zhang.miR-761 inhibits tumor progression by targeting MSI1 in ovarian carcinoma. Tumour Biol.2016; 37(4): 5437-5443.

57. Chen H, Liu J, Wang H, Cheng Q, Zhou C, Chen X, et al. Inhibition of RNABinding Protein Musashi-1 Suppresses Malignant Properties and Reverses Paclitaxel Resistance in Ovarian Carcinoma. J Cancer. 2019;10(6):1580-92.

58. Kang SH, Cho J, Jeong H, Kwon SY. High RNA-binding Motif Protein 3 Expression Is Associated with Improved Clinical Outcomes in Invasive Breast Cancer. J Breast Cancer. 2018;21(3):288-96.

59. Ye F, Jin $P$, Cai $X, C$ ai $P$, Cai H. High RNA-Binding Motif Protein 3 (RBM3) Expression is Independently Associated with Prolonged Overall Survival in Intestinal-Type Gastric Cancer. Med Sci Monit. 2017;23:6033-41.

60. Ehlén $\AA$, Nodin B, Rexhepaj E, Brändstedt J, Uhlén M, Alvarado-Kristensson $M$, et al. RBM3-regulated genes promote DNA integrity and affect clinical outcome in epithelial ovarian cancer. Transl Oncol. 2011:4(4):212-21.

61. Siesing, C., H. Sorbye, A. Dragomir, P. Pfeiffer, C. Qvortrup, F. Pontén, et al. High RBM3 expression is associated with an improved survival and oxaliplatin response in patients with metastatic colorectal cancer. PLoS One.2017; 12(8): e0182512.

62. Jonsson L, Gaber A, Ulmert D, Uhlén M, Bjartell A, Jirström K. High RBM3 expression in prostate cancer independently predicts a reduced risk of biochemical recurrence and disease progression. Diagn Pathol. 2011;6:91.

63. Ehlén A, Brennan DJ, Nodin B, O'Connor DP, Eberhard J, Alvarado-Kristensson $\mathrm{M}$, et al. Expression of the RNA-binding protein RBM3 is associated with a favourable prognosis and cisplatin sensitivity in epithelial ovarian cancer. J Transl Med. 2010;8:78.

64. Xu Z, Li X, Li H, Nie C, Liu W, Li S, et al. Suppression of DDX39B sensitizes ovarian cancer cells to DNA-damaging chemotherapeutic agents via destabilizing BRCA1 mRNA. Oncogene. 2020;39(47):7051-62. 
Publisher's Note

Springer Nature remains neutral with regard to jurisdictional claims in published maps and institutional affiliations.
Ready to submit your research? Choose BMC and benefit from:

- fast, convenient online submission

- thorough peer review by experienced researchers in your field

- rapid publication on acceptance

- support for research data, including large and complex data types

- gold Open Access which fosters wider collaboration and increased citations

- maximum visibility for your research: over $100 \mathrm{M}$ website views per year

At BMC, research is always in progress.

Learn more biomedcentral.com/submissions 